\title{
Surface Assisted Laser Desorption lonization- Mass Spectrometry (SALDI-MS) for Analysis of Polyester Degradation Products
}

\author{
Nina Aminlashgari, Minna Hakkarainen \\ Department of Fibre and Polymer Technology, School of Chemical Science and Engineering, KTH Royal Institute of Technology, \\ Stockholm, Sweden
}

\begin{abstract}
Novel surface assisted laser desorption ionization-mass spectrometry (SALDI-MS) method was developed for rapid analysis of low molecular mass polyesters and their degradation products by laser desorption ionization-mass spectrometry. Three polycaprolactone materials were analyzed by the developed method before and after hydrolytic degradation. The signal-to-noise values obtained by SALDI-MS were 20-100 times higher compared with the ones obtained by using traditional MALDI-MS matrices. A clean background at low mass range and higher resolution was obtained by SALDI-MS. Different nanoparticle, cationizing agent, and solvent combinations were evaluated. Halloysite nanoclay and magnesium hydroxide showed the best potential as SALDI surfaces. The SALDI-MS spectrum of the polyester hydrolysis products was verified by ESI-MS. The developed SALDI-MS method possesses several advantages over existing methods for similar analyses.
\end{abstract}

Key words: Surface assisted laser desorption ionization-mass spectrometry (SALDI-MS), Polyester, Polycaprolactone, Degradation, Nanoparticles

\section{Introduction}

$\mathrm{M}$ olecular level characterization of degradable polymers and their degradation products is crucial for successful use of these materials in packaging and biomedical applications [1]. Aliphatic polyesters such as poly( $\varepsilon$-caprolactone) (PCL), polylactide (PLA), and polyglycolide (PGA) are known to undergo hydrolytic degradation. Typical tools for analysis of degradation products are electrospray ionizationmass spectrometry (ESI-MS) [2, 3], LC-MS [4], and GC-MS $[5,6]$. However, the complex degradation matrices, together with polarity, water solubility, and large molecular mass range, still present significant analytical challenges. Mass spectrometric tools are ideal for detection and understanding of the degradation pathways of macromolecules [7].

Matrix assisted laser desorption ionization-mass spectrometry (MALDI-MS) is a routine tool for detection of high molecular weight polymers. In its traditional form, it has low

Correspondence to: Minna Hakkarainen; e-mail: minna@polymer.kth.se utility for analysis of low molecular mass compounds below $1000 \mathrm{Da}$ due to matrix related ions that appear in the low mass range and mask the signals of interest. A modification of MALDI-MS is a technique called surface assisted laser desorption ionization-mass spectrometry (SALDI-MS), where a surface is applied that absorbs laser energy and transfers it to the analyte molecules in the same way as the traditional low molecular mass matrix [8]. However, the surface does not interfere with the analyte signals and a clean spectrum can be obtained in low mass range. Nanoparticles have been used as surfaces for LDI because of their high surface area, high photo-absorption at the working laser band, and low heat capacity [9]. Nanoparticles are popular surfaces for screening of drugs, protein, and peptides [10, 11]. Porous polymer monoliths [12] and oxidized carbon nanotubes [13] are also excellent SALDI surfaces. Solventfree MALDI is an alternative method to SALDI-MS with a quick and easy applicability [14, 15].

Polyester hydrolysis products present a great analytical challenge due to polarity, low volatility, and large 
molecular mass range. We evaluated the potential of SALDI-MS as an analytical tool for screening these compounds. Method development was carried out by utilizing low molecular mass polycaprolactones as model compounds. The results were compared with conventional MALDI-MS analysis, and they were also verified by ESI-MS analysis of the same samples. In addition, a short-term hydrolysis study was performed to prove the applicability of the method in hydrolysis product analysis.

\section{Experimental}

\section{Materials}

Three polycaprolactone materials were purchased from Sigma Aldrich (Milwaukee, USA), polycaprolactone triol with average molecular weight $900 \mathrm{gmol}^{-1}$ (PCL900) according to Sigma Aldrich specifications and two polycaprolactone diols with average molecular weight of $1250 \mathrm{gmol}^{-1}$ (PCL1250) and $2000 \mathrm{gmol}^{-1}$ (PCL2000), see Figure 1 for structural information.

\section{Method Development}

The method development was carried out by analyzing PCL900 with different nanoparticle, solvent, and cationizing agent combinations. The nanoparticles were halloysite nanoclay, magnesium oxide, titanium dioxide, and silicon nitride (Sigma Aldrich) and graphitized carbon black (Lara laboratory analitici di ricerca associati, Rome, Italy). The solvents were HPLC grade tetrahydrofuran (THF, VWR), chloroform, and acetone (Fisher Scientific, Loughborough, UK). The concentration of nanoparticles in THF, chloroform and acetone was $20 \mathrm{gL}^{-1}$. The cationizing agents employed were sodium iodide (NaI) (Sigma Aldrich) $\left(1 \mathrm{gL}^{-1}\right.$ or $10 \mathrm{gL}^{-1}$ in THF), formic acid (0.1\% in 50:50 THF: $\mathrm{H}_{2} \mathrm{O}$ ), and trifluoroacetic acid (TFA) (Sigma Aldrich) (0.1\% in THF). The concentration of PCL900 used for the method development was $2 \mathrm{gL}^{-1}$ in THF.

The surface, solvent, and cationizing agent that gave best results were chosen and employed for further analysis of the remaining two polyesters and for the analysis of the degradation products from all three polyesters.

\section{Hydrolysis}

Approximately $50 \mathrm{mg}$ of the polymer was dissolved in $5 \mathrm{~mL}$ $\left(10 \mathrm{mg} \mathrm{mL} \mathrm{m}^{-1}\right)$ LC-MS grade water (Merck, Darmstadt, Germany). The samples were placed in $20 \mathrm{~mL}$ glass vials and sealed with septa for exposure in an oven at 37 and $60{ }^{\circ} \mathrm{C}$. After $7 \mathrm{~d}$, the samples were withdrawn from the vials and the water fractions were prepared for analysis.

\section{Surface Assisted Laser Desorption Ionization- Mass Spectrometry (SALDI-MS)}

The SALDI-MS analysis of polycaprolactone before and after degradation was performed using a Bruker UltraFlex time-of-flight (TOF) mass spectrometer with a SCOUTMTP ion source (Bruker Daltonics, Bremen, Germany) in reflector mode, equipped with a nitrogen laser $(337 \mathrm{~nm})$. For the degradation product analysis, the water fraction was evaporated and the residues were dissolved in $1 \mathrm{~mL}$ THF. The polycaprolactone before degradation was dissolved in THF at the same concentration, $10 \mathrm{mg} \mathrm{mL}^{-1}$. Nanoparticles were suspended in different solvents and solutions of cationizing agents were made. For fast evaporation of the three components, sample, surface, and cationizing agent solutions were spotted separately on the target plate with the volume ratios 3:1:1 $\mu \mathrm{L}$. The mass-to-charge $(\mathrm{m} / \mathrm{z})$ ratio range was 200-2000 with reflector voltage of $26.3 \mathrm{kV}$ and accelerated voltage of $25 \mathrm{kV}$. The spectrum obtained for each sample is an accumulation of 10 spectra with 200 laser shots at 10 different spots.

\section{Matrix Assisted Laser Desorption Ionization-Mass Spectrometry (MALDI-MS)}

For the MALDI analysis, 2,5-dihydroxybenzoic acid (DHB) (Fluka, Buchs, Switzerland), 2-(4-hydroxyphenylazo)benzoic acid (HABA) (Fluka) and 1,8,9-antracenotriol (dithranol) (Fluka, Buchs, Switzerland), was used as matrix and $\mathrm{NaI}$ as cationizing agent. The matrix was dissolved in THF to a concentration of $10 \mathrm{gL}^{-1}$ and mixed with the sample and cationizing agent (same concentration as earlier) at a volume ratio of 10:1:1 $\mu \mathrm{L}$ respectively and $1 \mu \mathrm{L}$ of that solution was spotted on the target plate.

\section{Electrospray Ionization-Mass Spectrometry (ESI-MS)}

The water fraction after hydrolysis was analyzed with a Finnigan LCQ ion trap mass spectrometer (Finnigan, San Jose, CA). LC-MS grade Methanol (Fluka) was added to the water solution containing the low molecular mass fraction to a volume ratio of $2: 1 \mathrm{vol} / \mathrm{vol}$ (water $/ \mathrm{MeOH})$. The solution was continuously infused into the ESI source by a syringe pump at a rate of $5 \mu \mathrm{L} / \mathrm{min}$. All experiments were run with positive ion mode. The LCQ ion source was operating at $5 \mathrm{kV}$ and the capillary heat was set to $175^{\circ} \mathrm{C}$. Nitrogen was employed as nebulizing gas and helium was used as damping and collision gas in the mass analyzer. No cationizing agents were added.

\section{Results and Discussion}

A SALDI-MS method was developed for screening of polyester degradation product patterns. These patterns cannot be analyzed by GC-MS due to high polarity, low 
(a)<smiles>[3H][13CH2]OCCCCCC(=O)OCCOCCOC(C)=O</smiles>

(b)<smiles>[R]OCC(CC)(CO[R])CO[R]</smiles>

Figure 1. Chemical structures of (a) the diols PCL1250 and PCL2000 and (b) the triol PCL900

volatility, and rather wide molecular mass range. On the other hand, the still relatively low molecular mass disturbs the analysis by traditional MALDI-MS. The obtained SALDI-MS spectra were compared with conventional MALDI-MS with DHB, HABA, and dithranol as matrix. The results were also verified by ESI-MS analysis of the degradation products.

\section{Method Development}

Different nanoparticles, such as titanium dioxide, halloysite nanoclay, graphitized carbon black, silicon nitride, and magnesium oxide, were suspended in diverse solutions, and nanoparticles that yielded the best signal response for PCL900 were determined. At first, solutions of nanoparticles

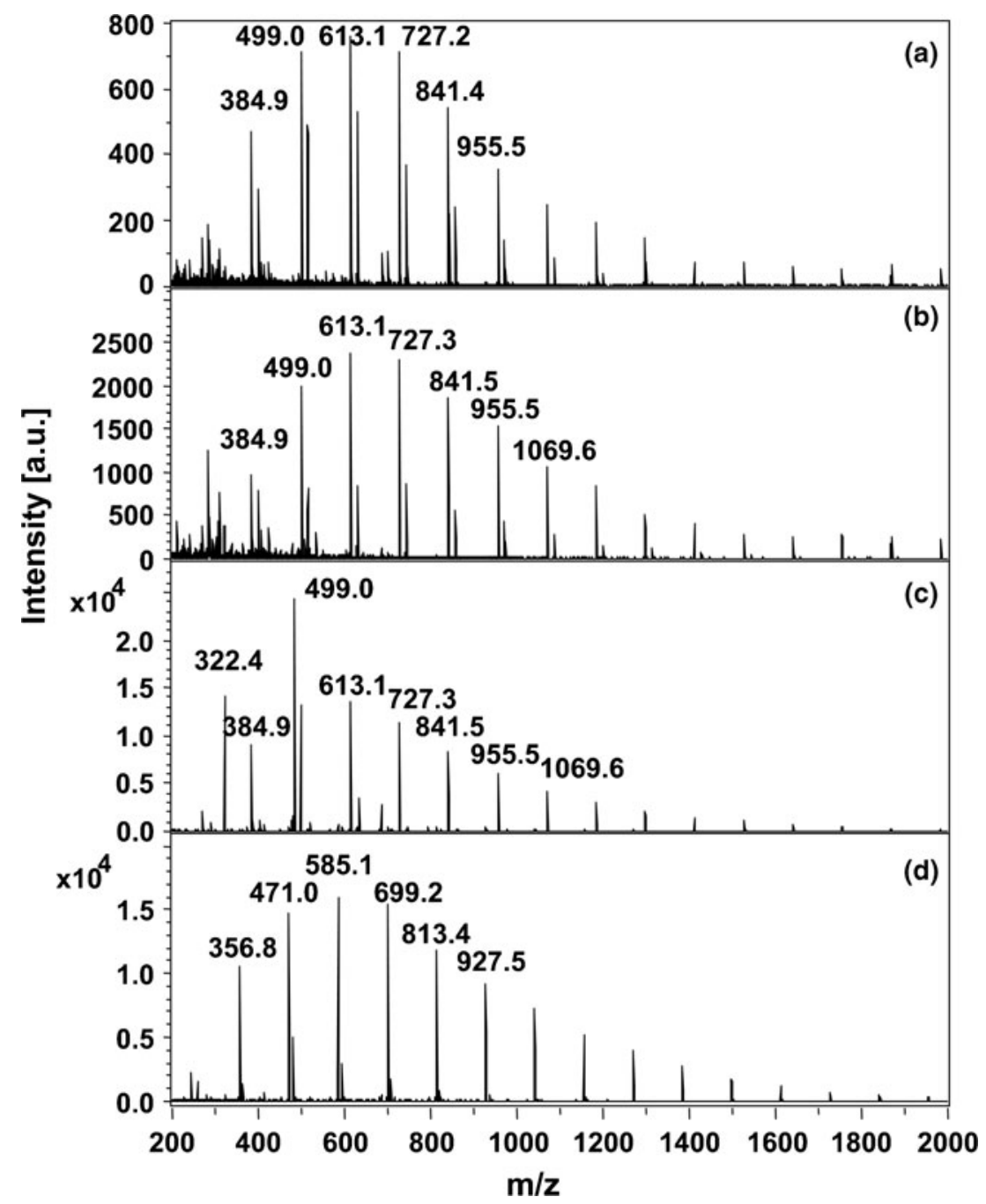

Figure 2. Spectra of PCL900 obtained with halloysite nanoclay as surface and (a) $0.1 \%$ TFA, (b) $0.1 \%$ formic acid, (c) Nal $10 \mathrm{mg} / \mathrm{mL}$ as cationizing agent, and (d) PCL1250 before degradation with halloysite nanoclay as surface and $\mathrm{Nal} 10 \mathrm{mg} / \mathrm{mL}$ as cationizing agent 


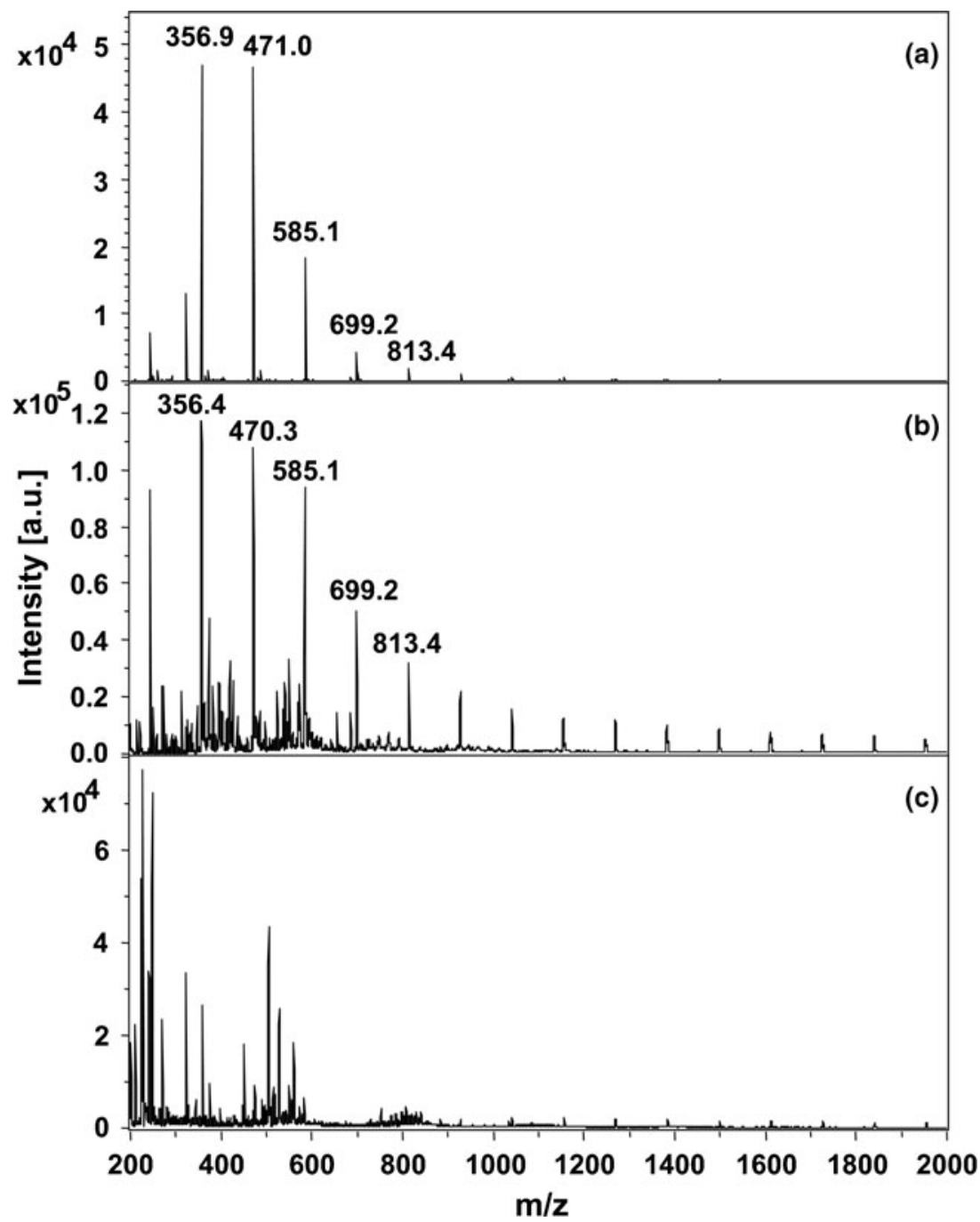

Figure 3. (a) SALDI-MS of PCL1250 after degradation with halloysite nanoclay as surface and (b) MALDI-MS of PCL1250 after degradation with DHB as matrix, (c) MALDI-MS of PCL1250 after degradation with dithranol as matrix

were spotted on the target plate to assure homogeneous distribution. Aggregation of nanoparticles was clearly seen for silicon nitride and titanium dioxide nanoparticles; they were therefore excluded from further method development. A fast evaporation method was chosen for the sample preparation on the target plate; the order of spotting each component on the stainless steel plate was first the surface, then the analytes, and finally the cationizing agent. Graphitized carbon black nanoparticles floated away in the edges of the spots. Halloysite nanoclay or the magnesium oxide was therefore chosen for further method development to keep the method as simple as possible. Furthermore, $3 \mu \mathrm{L}$ of $20 \mu \mathrm{g} \mu \mathrm{L}^{-1}$ nanoparticle solution was sufficient to cover a whole spot on the stainless steel plate. A higher concentration of nanoparticles did not improve the results, which is in agreement with our earlier study, where nanocomposites were used as SALDI surfaces [16].

Trifluoroacetic acid (TFA), sodium iodide, and formic acid were tested as cationizing agents, and a comparison of spectra obtained for PCL900 by utilizing different cationizing agents is shown in Figure 2. Sodium iodide resulted in highest signal intensities for PCL900. Both halloysite nano-

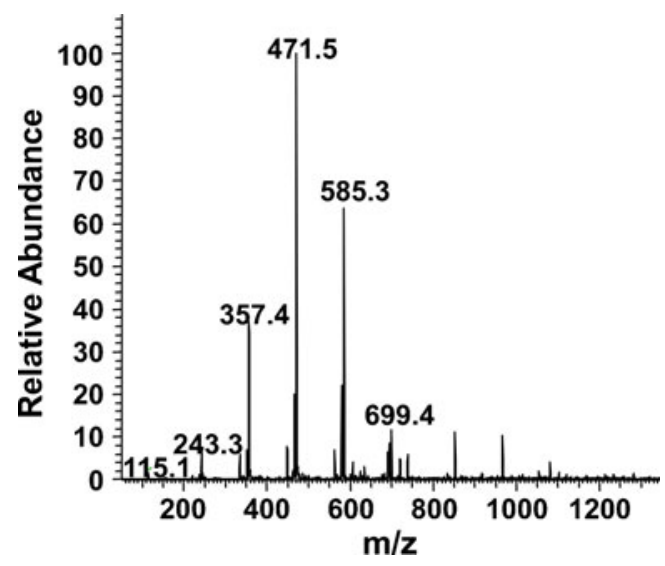

Figure 4. ESI-MS of PCL1250 hydrolysis products 
clay and magnesium oxide showed good potential as surfaces for polyester analysis.

\section{SALDI-MS Analysis of Hydrolysis Products}

The developed method was employed for analysis of degradation products of PCL900, PCL1250, and PCL2000. In Figure 2d, the SALDI-MS spectrum of PCL1250 before degradation is shown with caprolactone-diol oligomers with up to 16 mer. The background is clean without disturbing ions even at the mass range $<1000 \mathrm{Da}$, which corresponds to the monomer up to heptamer. The most intense peak observed was caprolactone tetramer with $\mathrm{S} / \mathrm{N}$ ratio of 1000 and $m / z=585.1$. The peak distribution in the SALDI-MS spectrum of non-hydrolyzed PCL1250 correlated closely with peak-distribution in MALDI-MS spectrum with DHB matrix. In both cases, oligomers with up to 16 mer were detectable. The spectrum of PCL1250 hydrolysis products is shown in Figure 3a. In comparison with the spectrum of unaged sample in Figure 2d, the formation of shorter oligomers during the hydrolysis is clearly seen, and the most intense signals correspond to caprolactone-diol dimer and trimer. Even in this case, clean background spectrum was obtained. Conversely, the MALDI-MS of the same sample shown in Figure $3 \mathrm{~b}$ and $\mathrm{c}$ demonstrated a noisy background in the low mass range $<500 \mathrm{Da}$, where the hydrolysis products should appear. In our model, study the concentrations were relatively high and the polymer degradation products could still be distinguished from the background noise using DHB as matrix. However, the other traditional matrices, dithranol (Figure 3c), and HABA (results not shown) resulted in very poor spectra. Analysis of unknown samples or samples with lower analyte concentration would be quite difficult by MALDI-MS because of these interfering matrix cluster ions.

The signal-to-noise $(\mathrm{S} / \mathrm{N})$ ratios for the shorter oligomers from monomer to heptamer were much higher when nanoparticle surfaces were used as targets for detection of degradation products instead of DHB matrix. In the upper mass range from heptamer to 11 mer, the surface still resulted in higher $\mathrm{S} / \mathrm{N}$ values; however, the differences in $\mathrm{S} /$ $\mathrm{N}$ ratios were smaller. Overall, the SALDI-MS approach was successful for the analysis of low molecular mass caprolactone oligomers and, compared with the best traditional matrix tested, up to 20 times higher $\mathrm{S} / \mathrm{N}$ values were obtained. In addition, the SALDI-MS method had higher mass resolution for the detection of caprolactone oligomers compared with the MALDI-MS method.

\section{Verification by ESI-MS}

The degradation products in the water fractions were analyzed by ESI-MS to verify the SALDI-MS results. Figure 4 shows the ESI-MS of PCL1250 degradation products. The most abundant peaks were dimer, trimer, and tetramer, and the obtained mass spectrum shows good correlation with the SALDI-MS mass spectrum. The SALDI-MS method has, however, some advantages. Buffer solutions are often applied for aging of biomedical materials to simulate the conditions in the human body. An ESI-MS analysis of buffer solutions requires a LC separation prior to the ESI analysis. SALDI-MS on the other hand tolerates buffer solutions and there is no need for a clean-up step or additional sample preparation.

\section{Conclusions}

Novel SALDI-MS method was developed to allow rapid screening of low molecular mass polyesters and their degradation product patterns by regular MALDI-MS instrument. Halloysite nanoclay and magnesium oxide showed good potential as SALDI surfaces for the analysis of degradation products. Compared with conventional MALDI-MS, the resolution was better and there was reduced background noise without interfering peaks in the low mass range. In addition, the intensity of the sample peaks and the signal-to-noise ratios obtained by SALDI-MS were significantly higher. The developed method exhibits several advantages compared with the currently used GCMS, LC-MS, and ESI-MS methods in the analysis of polymer degradation products as it reduces sample preparation and expands the range of possible analytes and sample matrixes.

\section{References}

1. Hakkarainen, M.: Aliphatic polyesters: abiotic and biotic degradation and degradation products. Adv. Polym. Sci. 157, 113-138 (2002)

2. Hakkarainen, M., Adamus, G., Höglund, A., Kowalczuk, M., Albertsson, A.-C.: ESI-MS reveals the influence of hydrophilicity and architecture on the water-soluble degradation product patterns of biodegradable homo- and copolyesters of 1,5-dioxepan-2-one and e-caprolactone. Macromolecules 41, 3547-3554 (2008)

3. Andersson, S.R., Hakkarainen, M., Inkinen, S., Södergård, A., Albertsson, A.-C.: Polylactide stereocomplexation leads to higher hydrolytic stability but more acidic hydrolysis product pattern. Biomacromolecules 11, 1067-1073 (2010)

4. Song, J., Šišková, A., Simons, M.G., Kowalski, W.J., Kowalczuk, M. M., van den Brink, O.F.: LC-multistage mass spectrometry for the characterization of Poly(Butylene Adipate-co-Butylene Terephthalate) copolyester. J. Am. Soc. Mass Spectrom. 22, 641-648 (2011)

5. Hakkarainen, M., Höglund, A., Odelius, K., Albertsson, A.-C.: Tuning the release rate of acidic degradation products through macromolecular design of caprolactone-based copolymers. J. Am. Chem. Soc. 129, 6308-6312 (2007)

6. Hakkarainen, M., Albertsson, A.-C.: Heterogenous biodegradation of polycaprolactone - low molecular weight products and surface changes. Macromol. Chem. Phys. 203, 1357-1363 (2002)

7. Aminlashgari, N.; Hakkarainen, M.: Emerging mass spectrometric tools for analysis of polymers and polymer additives. Adv. Polym. Sci. (2011), in press.

8. Tanaka, K., Waki, H., Ido, Y., Akita, S., Yoshida, Y., Yoshida, T., Matsuo, T.: Protein and polymer analyses up to $\mathrm{m} / \mathrm{z} 100000$ by laser ionization time-of-flight mass spectrometry. Rapid Commun. Mass Spectrom. 2, 151-153 (1988)

9. Amini, N., Shariatgorji, M., Thorsén, G.: SALDI-MS signal enhancement using oxidized graphitized carbon black nanoparticles. J. Am. Soc. Mass Spectrom. 20, 1207-1213 (2009)

10. Su, C.-L., Tseng, W.-L.: Gold nanoparticles as assisted matrix for determining neutral small carbohydrates through laser desorption/ 
ionization time-of-flight mass spectrometry. Anal. Chem. 79, 16261633 (2007)

11. Watanabe, T., Kawasaki, H., Yonezawa, T., Arakawa, R.: Surfaceassisted laser desorption/ionization mass spectrometry (SALDI-MS) of low molecular weight organic compounds and synthetic polymers using zinc oxide ( $\mathrm{ZnO})$ nanoparticles. J. Mass Spectrom. 43, 1063-1071 (2008)

12. Peterson, D.S., Luo, Q., Hilder, E.F., Svec, F., Fréchet, J.M.J.: Porous polymer monolith for surface-enhanced laser desorption/ionization time-of-flight mass spectrometry of small molecules. Rapid Commun. Mass Spectrom. 18, 1504-1512 (2004)

13. Pan, C., Xu, S., Hu, L., Su, X., Ou, J., Zou, H., Guo, Z., Zhang, Y., Guo, B.: Using oxidized carbon nanotubes as matrix for analysis of small molecules by MALDI-TOF MS. J. Am. Soc. Mass Spectrom. 16 883-892 (2005)

14. Trimpin, S., Rouhanipour, A., Az, R., Räder, H.J., Müllen, K.: New aspects in matrix-assisted laser desorption/ionization time-of-flight mass spectrometry: a universal solvent-free sample preparation. Rapid Commun. Mass Spectrom. 15, 1364-1373 (2001)

15. Trimpin, S., Wijerathne, K., McEwen, C.N.: Rapid methods of polymer and polymer additives identification: multi-sample solvent-free MALDI, pyrolysis at atmospheric pressure, and atmospheric solids analysis probe mass spectrometry. Anal. Chim. Acta 654, 20-25 (2009)

16. Aminlashgari, N., Shariatgorji, M., Ilag, L.L., Hakkarainen, M.: Nanocomposites as novel surfaces for laser desorption ionization mass spectrometry. Anal. Methods 3, 192-197 (2011) 